\title{
Under-appreciated and Overlooked Modes of Exercises on Key Vascular Functions
}

Author
Hirofumi Tanaka

\section{Affiliation}

Department of Kinesiology and Health Education, The University of Texas at Austin, Austin TX USA

\section{Key words}

aging, arterial stiffness, endothelial function, physical activity

accepted 14.09.2020

published online 19.10 .2020

Bibliography

Int J Sports Med 2022; 43: 586-592

DOI $10.1055 / a-1268-8607$

ISSN $\quad 0172-4622$

(c) 2020. Thieme. All rights reserved.

Georg Thieme Verlag KG, Rüdigerstraße 14, 70469 Stuttgart, Germany

\section{Correspondence}

Dr. Hirofumi Tanaka

Department of Kinesiology and Health Education, The University of Texas at Austin,

2109 San Jacinto Blvd,

Austin, TX 78712

United States

Tel.: +15122324801, Fax: +15124710946

htanaka@austin.utexas.edu

\begin{abstract}
The arterial system has two primary functions. The conduit function is to transport adequate supply of oxygen and nutrients to the tissues, and the cushioning function is to buffer and cushion the pulsatile pressure exerted by intermittent ventricular contractions. The impairments in these two functions often result from physiological changes characterized by endothelial dysfunction and arterial stiffening. Habitual physical exercise has been advocated to combat these physiological dysfunctions. However, exercise is remarkably diverse, as it can be performed in different media (water, land or snow), seasons (winter or summer), and settings (individual, pair or team). In contrast to mainstream modes of exercise including walking and running, many of the alternative or "minor" forms of exercise have been under-researched by investigators in research fields and overlooked by clinicians and practitioners in clinical settings. It remains largely unknown whether these alternative forms of exercise are associated with favorable changes in arterial stiffness and endothelium-dependent vasodilation. The current review introduces and summarizes research investigations that evaluated the impacts of these under-appreciated and overlooked exercises and their impacts on key markers of vascular functions in humans.
\end{abstract}

\section{Introduction}

Cardiovascular disease remains the number one cause of death in most industrialized countries [1]. Cardiovascular disease is a cluster of various types of circulatory diseases affecting the heart and vasculatures. When breaking it down, the majority of cardiovascular diseases, commonly referred to as "heart diseases," are diseases of arteries, as the pathogenesis of coronary "artery" disease, stroke or cerebrovascular accident, high blood pressure, and peripheral "artery" disease is attributed to arterial circulations. Despite this, none of the traditional risk factors for cardiovascular disease address arterial functions that precede the manifestation of cardiovascular disease [2]. The vascular system is critical for health and survival of all tissues and organs, as the vast network perfuses oxygen and nutrients and eliminates waste products. During exercise, marked increases in systemic blood flow (i. e. cardiac output) and redistribution of blood flow are supported by the cushioning or buffering functions of arteries that are characterized by arterial stiffness [3]. Endothelial function plays a critical role in regulating amounts of perfusion to various tissues and organs during exercise [4].

Regular physical exercise confers benefits on a number of different diseases and conditions and acts to reduce overall mortality [5]. One of the most striking conditions on which regular exercise is shown to exert its influence is cardiovascular disease [6]. The association of regular physical activity and cardiovascular mortality is well documented in both observational and longitudinal studies [5]. However, the exact mechanisms underlying the effects of habitual exercise on cardiovascular disease are currently unknown but have often been attributed to improvements in cardiovascular risk factors [7]. However, the proportion of the beneficial effects of regular exercise that can be explained by improvements in cardiovascular risk factors is fairly small [8]. In one study, $>60 \%$ of the variance in coronary heart disease reduction by exercise was not 
explained by a comprehensive set of seven traditional risk factors [8]. One of the arguments that has been raised is that improvements in arterial functions could explain a large remaining proportion of the association [9-11].

There are a number of arterial functions being measured in clinical and research settings [12]. The focus of the present review is placed on two key subclinical markers of vascular diseases: arterial stiffness and endothelial function. These two functions are also two primary phenotypic features of vascular aging [11]. Large elastic arteries in cardiothoracic and carotid regions expand and recoil during cardiac contraction and relaxation, thereby damping and cushioning fluctuations in arterial pressure and blood flow. The stiffening of large elastic arteries impairs the buffering function of the arterial system and leads to cardiovascular dysfunction via a variety of mechanisms, including elevated systolic blood pressure, augmented left ventricular afterload, decreased coronary blood flow, and blunting of arterial baroreflex sensitivity. Indeed stiffness of central elastic arteries is associated with cardiovascular outcomes above and beyond cardiovascular risk factors [13]. In contrast to central elastic arteries, stiffness of peripheral muscular arteries (e. g. brachial artery, femoral artery) does not change with aging or with disease states and does not predict cardiovascular risks $[14,15]$. Moreover, this segment of arterial stiffness does not appear to change with exercise training $[16,17]$. Accordingly, clinical and functional significance of peripheral artery stiffness is currently uncertain.

Endothelium-dependent vasodilation can also be divided into macrovascular (conduit artery) and microvascular (resistance artery) measures and are typically evaluated using brachial artery flow-mediated dilation and forearm blood flow responses to pharmacological stimuli (e. g. acetylcholine and bradykinin) [12] in humans. Interestingly, these two measures of endothelium-dependent vasodilation are influenced differentially by age and are not related to one another [18]. Nevertheless, endothelial function plays a pivotal role in all aspects of atherosclerosis and has predictive value for future cardiovascular events [19]. The amount of available studies on venous function is extremely limited and not included in the present review. However, there is experimental evidence indicating that venous compliance in legs is greater in endurancetrained adults than in sedentary adults [20] and increases with endurance training interventions [21].

Various forms of exercise intervention have been conducted and evaluated to reduce risks of cardiovascular disease. Most of the available studies to date have addressed effects of walking and running as an aerobic exercise and weight training as a form of resistance training on vascular functions. A number of review papers have been written and are available in the literature to emphasize the benefits or a lack thereof of these traditional or main stream exercises on the vasculature [9-11]. However, exercises and sporting activities are extremely diverse, as a substantial variation is introduced every time it is practiced in different media (water, land or snow), seasons (winter or summer), and settings (individual, pair, group or team) (॰ Fig. 1).

A myriad of choices among various modes of exercise to target vascular dysfunction may be analogous to pharmacological treatments for hypertension. There are a total of six different classes of antihypertensive drugs being used clinically [22]. Even though the initial treatment strategy is diuretics for most, clinicians are advised to change the choice of drugs depending on a number of conditions including coexisting disease, race, and side effects [23]. Even within the class of diuretics, there are multiple choices of drugs to select. As a choice of proper drug is critically important to elucidate the best possible clinical outcome, a selection of proper and preferred exercise mode that can be adhered to is equally important to enhance the effectiveness of exercise programs. Participants assigned to aerobic exercise class that matched their preferred choice had better attendance and expressed a greater intention to continue exercising than those assigned to the standard exercise class [24]. If walking or running is not a preferred mode of exercise, which activity will be selected as a primary form of exercise? If the overall exercise program is to be diversified to reduce boredom and enhance enjoyment, as well as to accommodate exercise tolerance, what other activity can be chosen to reduce cardiovascular risks? More importantly, investigators and clinicians should be aware of the research evidence for a variety of exercises used to enhance vascular functions.

In this review, four categories of exercises and sports that have been under-appreciated and overlooked are highlighted ( $\triangleright$ Fig. 1). They include water-based exercises (swimming, aquatic exercise, rowing), mind-body interaction exercises (yoga, Tai Chi, walking meditation), ball sports (soccer, football), and winter sports (Alpine skiing). For the purpose of this review, only investigations that reported arterial stiffness and endothelial function as outcome measures are included in the review.

\section{Swimming}

Swimming is one of the most popular forms of physical activity, and is often included as a recommended mode of exercise for overall health and fitness [25]. Similar to walking and cycling, it is a dynamic rhythmic aerobic exercise that involves a large muscle mass [26]. But swimming is substantially different as a mode of exercise. It is performed in a different medium than walking/running (i.e. water) and in the supine/prone, rather than the upright posture,

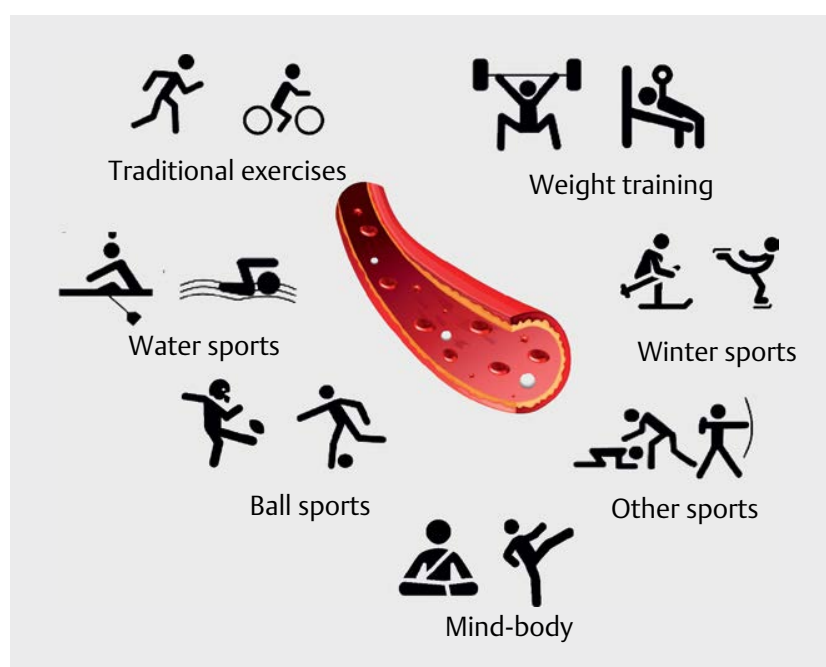

Fig. 1 Influence of a variety of exercises and sporting activities on vascular function. 
resulting in a zero-gravity situation. Because of its non-weightbearing nature, swimming has a much lower rate of orthopedic injury than does running [27]. Indeed swimming appears to be an excellent form of exercise for patients with osteoarthritis [28]. Due to decreased heat load resulting from high thermoconductivity of water, risks of heat exertion that obese adults often experience are reduced substantially. Unlike other popular modes of exercises (e. g. walking, cycling), swimming is a full-body task that the majority of the propulsive force comes primarily from upper body [29]. As differences in vasodilatory responses have been reported between arms and legs [30], arterial adaptations may be different between swimming and land-based exercises. From the exercise prescription standpoint, swimming can be an ideal form of exercise, particularly for elderly patients, obese patients, patients with arthritis, and those who have problems and tolerance issues with weight-bearing activities. In spite of the fact that swimming is one of the most popular, most practiced and most prescribed modes of exercise, surprisingly little research-related information is available regarding the influence of regular swimming on cardiovascular risks [25].

In a cross-sectional study, middle-aged and older swimmers demonstrate reduced levels of arterial stiffness than sedentary peers and a similar level of arterial stiffness to runners who are matched for weekly training volume [31]. However, brachial artery flow-mediated dilation of swimmers is similar to sedentary peers and lower than runners [31]. This particular finding seems strange at first glance as the major working muscles in swimming are arms on which flow-mediated dilation was measured. From the standpoint of redistribution of blood flow during exercise, blood flow to non-working muscles (arms) is thought to be reduced [32]. However, substantial increases in arm blood flow have been observed during leg cycling exercise [33].

In an exercise intervention study involving older patients with osteoarthritis, swimming training performed at moderate intensity reduced central arterial stiffness as assessed by carotid-femoral pulse wave velocity and increased endothelium-dependent vasodilation as evaluated by brachial artery flow-mediated dilation [34]. Cycling training, which was implemented as a non-weight bearing land-based comparison group, decreased arterial stiffness but did not change endothelium-dependent vasodilation [34]. Physiological mechanisms underlying the impact of swimming training on vascular function is not known. But in an animal study, reductions in arterial stiffness induced by swimming exercise are associated with the corresponding changes in eNOS phosphorylation [35].

In a clinical trial involving older patients with essential hypertension, three months of swimming intervention performed at moderate intensity not only reduced ambulatory and central blood pressure but also improved endothelium-dependent vasodilation and central arterial stiffness [36]. This finding is particularly noteworthy because walking-based exercise interventions have not been shown to be effective as destiffening therapy for older patients with essential hypertension and diabetes [37-39]. Taken together, the available evidence indicates that swimming is an excellent form of exercise to improve vascular functions in older adults with and without existing clinical conditions.

\section{Aquatic exercise}

Swimming is an excellent mode of physical activity. However, it requires adequate skills and techniques to achieve target exercise intensity during exercise. As such, some individuals cannot initiate the exercise program right away. Aquatic exercise is an alternative water-based exercise that most people can begin to engage without any special skills and techniques. Does aquatic exercise performed in shallow water in the upright posture produce similarly beneficial impact on vascular functions to swimming? Ten weeks of aquatic exercise reduces aortic pulse wave velocity in older adults [40]. In patients with type 2 diabetes, an improvement in flow-mediated dilation has been observed after eight weeks of aquatic exercise performed at a moderate intensity [41]. Thus, the available studies, though limited in numbers, are consistent with the notion that aquatic exercise is beneficial in improving vascular functions.

Considering that both swimming and aquatic exercise exert favorable influence on the vasculature, could at least some portion of the favorable influence be attributed to water immersion? Interestingly, there are many investigations indicating that exercises performed in water are more effective in improving vascular function than the same exercises performed on land. For example, in older patients with type 2 diabetes, land-based and water-based moderate cycling exercise performed three times a week for 12 weeks produced significant increases in flow-mediated dilation in the popliteal artery and decreases in arterial stiffness [42]. However, indices of cutaneous macrovascular reactivity as assessed by post-occlusive reactive hyperemia improved only in the waterbased exercise group [42]. The reason for the relative efficacy of water-based vs. land-based exercises is not clear. However, there is an experimental evidence indicating that circulating levels of nitric oxide metabolites increased after water-based exercise but not after same exercise on land in patients with heart disease [43]. Similarly, skeletal muscle nitric oxide synthase content was increased after 12 weeks of aquatic treadmill exercise but not after treadmill exercise performed on land [44]. Plasma and blood volume can exert marked influences on blood pressure and vascular function. Plasma volume expansion that occurs with exercise appears to be smaller in swimmers than in runners [45].

\section{Rowing}

Theoretically, rowing is an outstanding form of exercise that is low impact and dynamic in nature and involves a large muscle mass. Rowing is also a time-efficient mode of exercise as it simultaneously involves components of both aerobic endurance and muscular strength as demonstrated by rowers' large aerobic capacity and muscle strength [46]. In an cross-sectional study, central arterial compliance is greater and beta-stiffness index is lower in habitual rowers than in age-matched sedentary controls [16]. The reduced arterial stiffness in rowers is associated with a greater cardiovagal baroreflex sensitivity [16].

In a study of young elite rowers, brachial artery flow-mediated dilation assessed by MRI was not different between rowers and sedentary controls [47]. Interestingly, endothelium-independent vasodilation as assessed by nitroglycerine administration was significantly lower in rowers than in sedentary controls [47]. There is also a report of no differences in plasma nitrite and nitrate and endothe- 
lin-1 levels between older habitual rowers and sedentary controls [48]. Physiological explanations for these findings are not clear. But it could be interpreted as habitual rowing producing smooth muscle cell desensitization. We were not able to locate clinical trials or randomized control trial involving rowing training on vascular function.

\section{Mind-body interaction exercises}

Mind-body medicine in the form of meditation, relaxation and prayer has become popular in recent decades for the strategies to manage psychological stress and improve overall health $[49,50]$. To augment its effect, it can be incorporated and combined into a form of exercise including yoga and Tai Chi. A number of other forms of mind-body interaction exercises (e. g. laughter yoga, Buddhist walking meditation) have also been studied actively [51, 52].

Tai Chi is an ancient Chinese callisthenic exercise and martial art that combines a series of slow, relaxed and graceful movements with deep breathing techniques. In an observational study, older practitioners of Tai Chi demonstrated enhanced endothelium-dependent vasodilation in the skin vasculature as assessed by changes in laser Doppler flow in response to iontophorertic infusion of acetylcholine [53]. Additionally, in older patients with peripheral neuropathy, progressive Tai Chi practiced three times a week for 12 weeks produced a $25 \%$ increase in vascular reactivity index measured by fingertip digital thermal monitoring [54]. In a randomized control trial that compared 16 weeks of Tai Chi training and an educational program, elderly women assigned to Tai Chi were able to improve both large artery and small artery compliance as assessed by the HDI device [55]. Thus, there is sufficient evidence to indicate that Tai Chi is effective in improving vascular function.

Walking meditation or mindful walking is a widely practiced form of Buddhist practice that focuses on mind-body interactions. It incorporates the principles of traditional meditation performed in the sitting position, including breathing, awareness, concentration and relaxation, with rhythmic exercise of walking [52]. In a clinical trial investigating the comparative effects of Buddhist walking meditation and traditional walking in middle-aged and older patients with type 2 diabetes, flow-mediated dilation increased significantly in both training groups [56]. However, a reduction in arterial stiffness, as well as a decrease in hemoglobin A1c, were observed only in the Buddhist walking meditation group. These results suggest that spirituality-based exercise program is effective in producing a multitude of health benefits relevant to middle-aged and older patients with type 2 diabetes.

\section{Yoga}

Yoga is a practice that has been performed over 5,000 years in the Eastern part of the world, and it has become increasingly popular as a mode of exercise in the Western part of the world. Yoga is an attractive form of exercise from the vascular health standpoint when it is broken down into its elements (e. g. stretching, static exercise and meditation) [57]. One primary physical component of yoga is stretching. An inverse association has been reported between trunk flexibility and arterial stiffness [58, 59]. Stretching intervention has been shown to reduce arterial stiffness in previously sedentary adults [60]. Additionally, even though a variety of yoga postures evoke significant increases in blood pressure [59], yoga postures are characterized by static or isometric exercises that could act to reduce blood pressure and improve endothelial function [61]. Moreover, meditation incorporated in yoga could improve vascular function presumably through reductions in sympathetic vasoconstrictor tone [62].

In a cross-sectional study comparing yoga practitioners and sedentary peers, no significant differences were found in central arterial stiffness and brachial artery flow-mediated dilation [63]. Twelve weeks of yoga practices did not change arterial stiffness and flowmediated dilation in previously sedentary middle-aged adults [63]. A lack of effects of yoga does not appear to be an issue of short intervention length, as eight months of yoga intervention also fails to reduce arterial stiffness in middle-aged premenopausal women [64]. In contrast, a study in India compared yoga and brisk walking performed for $1 \mathrm{~h}$ six days a week for 12 weeks, and found that arterial stiffness was significantly reduced with yoga but not with brisk walking in elderly participants with elevated blood pressure [65]. Compared with regular or traditional yoga (e. g. hatha yoga), there is more evidence indicating the benefits of Bikram yoga, a highly standardized rigorous yoga of 26 postures practiced in a heated environment with $40-60 \%$ relative humidity. A relatively shortterm practice of Bikram yoga improved brachial artery flow-mediated dilation [66] and central arterial stiffness [67] although the effects seem to be modulated by age. Given the observations that both sauna [68] and hot water immersion [69] have been shown to improve vascular function, it seems reasonable to attribute the superior effects of Bikram yoga to the heated practice environment. However, the magnitude of improvements in endothelium-dependent vasodilation appears to be similar between Bikram yoga performed in the thermoneutral environment and hot humid environment [70].

\section{Ball sports}

Association football, more commonly referred to as soccer or football, is the most popular sport in the world. The playing style touches on many elements of overall physical fitness and can be considered as a form of high-intensity interval training incorporated into a ball game. Since it is played as a team with teammates, it is perceived as more motivating and enjoyable than individual exercise or sport [71]. A cross-sectional study demonstrated that older soccer players show more favorable endothelial function as measured by peripheral artery tonometry [72]. However, an exercise intervention study conducted by the same group is not consistent with the cross-sectional study. Twelve months of soccer training did not change endothelium-dependent vasodilation in older men although the overall cardiovascular benefits were confirmed by significant increases in maximal oxygen consumption [73]. In another intervention study in middle-aged men with hypertension, six months of soccer training performed twice a week reduced augmentation index [74], an index of arterial wave reflection that is indirectly associated with arterial stiffness. Given a small number of studies relative to the popularity of soccer, a lot more studies are needed to determine its vascular effects.

American-style football is the most popular spectator sport in the United States. American football has spread all over the world, as American football federations are present on most continents. Unlike other ball sports, this team sport is not widely practiced by 
middle-aged and older populations. However, non-contact variants of American football, including flag football and touch football, are practiced by youths, coeds and older adults. In collegiate football athletes, arterial stiffness increases significantly from a preseason to the end of a single season of football [75]. Additionally, arterial stiffness measured in the pre-season increases gradually year after year during the college football players' careers [76]. A key component of the overall training regimen in American-style football is strenuous resistance training. Resistance training has been demonstrated to increase arterial stiffness in young and middle-aged adults [77-79]. Stiffening effects of resistance training have also been observed in resistance training performed at a moderate intensity [80]. In studies involving older adults, resistance training does not appear to elevate arterial stiffness possibly due to the high baseline arterial stiffness causing a ceiling effect $[60,81]$.

Information regarding the effects of other ball sports on vascular function is very limited. For instance, tennis players are frequently-used experimental model of investigating dominant (active) and non-dominant (relatively inactive) limbs [82-84]. However, there have not been any randomized clinical trials or exercise interventions that addressed the effects of tennis practices on vascular function. Ball sports are extremely diverse in nature. A variety of ball sports including basketball and volleyball are practiced and enjoyed by youths and adults varying widely in age. More research emphasis should be devoted to these overlooked ball sports.

\section{Winter sports}

Winter sports are sports or recreational activities that are performed on snow or ice. Among them, Alpine skiing is practiced widely in mountainous regions that receive adequate amounts of snowfall in the winter. It is the most popular winter sport worldwide, and over a million people enjoy Alpine skiing in Austria alone. From the physiological standpoint, Alpine skiing can be considered a form of interval training as hard work on downhill slope that could stress both muscular strength and cardiovascular fitness is interspersed by respites on the chairlift or gondola. In a study involving older healthy adults who were either beginners or intermediate levels of Alpine skiing at the beginning of the study, 12 weeks of Alpine skiing did not alter circulating biomarkers of endothelial function and inflammation [85]. In a follow-up study [86], circulating biomarkers of endothelial function did not change once again. Additionally, the reactive hyperemia index did not change in the intervention group although early, but not late, endothelial progenitor cells increased [86]. Thus, the currently available information does not seem to support the notion that Alpine skiing exerts beneficial impact on endothelial function. We were not able to find clinical trials evaluating Alpine skiing or other winter sports on arterial stiffness. Even though a variety of other winter sports, including cross-country skiing, snow shoeing, ice climbing and curling are widely practiced and enjoyed recreationally, their impacts on vascular health are largely unknown.

\section{Perspective}

In spite of the fact that the modes of exercise and sports that individuals can select and engage in are numerous, most of the available research studies have focused on the mainstream exercises such as walking/running and resistance/weight training. There are a number of under-emphasized and overlooked exercises that can exert beneficial impacts on key vascular functions. For some exercises (e. g. archery, tennis), no research has been conducted to determine their efficacy. In real life situations, exercise is a part of the overall lifestyle modifications and often combines with other interventions. However, additive or synergistic effect of exercise combined with other lifestyle modifications are largely unknown. Individuals are advised to diversify exercise routines to reduce boredom and enhance enjoyment but the effects of "cross-training" that combine multiple modes of exercise have not been studied adequately [87]. Appreciation of the effects of under-appreciated and overlooked modes of exercise is critical, as the exercise prescription can be tailored or personalized to maximize the effectiveness of exercise therapy. Clearly, a lot more research on vascular function is warranted to line up as many exercise modes as we can for the "exercise is medicine" arsenal.

\section{Acknowledgment}

This manuscript complies with the Journal's ethical standards [88].

\section{Conflict of Interest}

The authors declare that they have no conflict of interest.

\section{References}

[1] Virani SS, Alonso A, Benjamin E] et al. Heart disease and stroke statistics-2020 update: A report from the American Heart Association. Circulation 2020; 141: e139-e596

[2] Liao D, Arnett DK, Tyroler HA et al. Arterial stiffness and the development of hypertension. The ARIC study. Hypertension 1999; 34: 201-206

[3] Tanaka H, Dinenno FA, Hunt BE et al. Hemodynamic sequelae of age-related increases in arterial stiffness in healthy humans. Am J Cardiol 1998; 82: 1152-1155

[4] Joyner M], Dietz NM. Nitric oxide and vasodilation in human limbs. J Appl Physiol (1985) 1997; 83: 1785-1796

[5] Blair SN, Kohl HW, Barlow CE et al. Changes in physical fitness and all-cause mortality. JAMA 1995; 273: 1093-1098

[6] Pearson TA, Blair SN, Daniels SR et al. AHA guidelines for primary prevention of cardiovascular disease and stroke: 2002 update: Consensus panel guide to comprehensive risk reduction for adult patients without coronary or other atherosclerotic vascular diseases. American Heart Association Science Advisory and Coordinating Committee. Circulation 2002; 106: 388-391

[7] Fiuza-Luces C, Santos-Lozano A, Joyner M et al. Exercise benefits in cardiovascular disease: Beyond attenuation of traditional risk factors. Nat Rev Cardiol 2018; 15: 731-743

[8] Mora S, Cook N, Buring JE et al. Physical activity and reduced risk of cardiovascular events: potential mediating mechanisms. Circulation 2007; 116: 2110-2118

[9] Tanaka H. Antiaging effects of aerobic exercise on systemic arteries. Hypertension 2019; 74: 237-243

[10] Green DJ, Spence A, Halliwill JR et al. Exercise and vascular adaptation in asymptomatic humans. Exp Physiol 2011; 96: 57-70 
[11] Seals DR, Desouza CA, Donato AJ et al. Habitual exercise and arterial aging. J Appl Physiol (1985) 2008; 105: 1323-1332

[12] Hartley C], Tanaka H. Assessment of macro- and microvascular function and reactivity. In: Naghavi M, Ed. Asymptomatic Atherosclerosis: Pathophysiology, Detection and Treatment. New York, NY: Humana Press; 2011: 265-278

[13] Willum-Hansen T, Staessen JA, Torp-Pedersen C et al. Prognostic value of aortic pulse wave velocity as index of arterial stiffness in the general population. Circulation 2006; 113: 664-670

[14] Meyer ML, Tanaka H, Palta P et al. Correlates of segmental pulse wave velocity in older adults: The atherosclerosis risk in communities (ARIC) study. Am J Hypertens 2016; 29: 114-122

[15] Kim ED, Ballew SH, Tanaka H et al. Short-term prognostic impact of arterial stiffness in older adults without prevalent cardiovascular disease. Hypertension 2019; 74: 1373-1382

[16] Cook JN, DeVan AE, Schleifer JL et al. Arterial compliance of rowers: Implications for combined aerobic and strength training on arterial elasticity. Am J Physiol Heart Circ Physiol 2006; 290: H1596-H1600

[17] Tanaka H, DeSouza CA, Seals DR. Absence of age-related increase in central arterial stiffness in physically active women. Arterioscler Thromb Vasc Biol 1998; 18: 127-132

[18] Eskurza I, Seals DR, DeSouza CA et al. Pharmacological vs. flowmediated assessments of peripheral vascular endothelial vasodilatory function in humans. Am J Cardiol 2001; 88: 47-49

[19] Veerasamy M, Bagnall A, Neely D et al. Endothelial dysfunction and coronary artery disease: a state of the art review. Cardiol Rev 2015; 23: $119-129$

[20] Monahan KD, Dinenno FA, Seals DR et al. Smaller age-associated reductions in leg venous compliance in endurance exercise-trained men. Am J Physiol Heart Circ Physiol 2001; 281: H1267-H1273

[21] Hernandez JP, Franke WD. Effects of a 6-mo endurance-training program on venous compliance and maximal lower body negative pressure in older men and women. J Appl Physiol (1985) 2005; 99 : 1070-1077

[22] Azizi M, Rossignol P, Hulot JS. Emerging drug classes and their potential use in hypertension. Hypertension 2019; 74: 1075-1083

[23] Whelton PK, Carey RM, Aronow WS et al. 2017 ACC/AHA/AAPA/ABC/ ACPM/AGS/APhA/ASH/ASPC/NMA/PCNA guideline for the prevention, detection, evaluation, and management of high blood pressure in adults: executive summary: A report of the American college of cardiology/American heart association task force on clinical practice guideline. Hypertension 2018; 71: 1269-1324

[24] Thompson CE, Wankel LM. The effects of perceived activity choice upon frequency of exercise behavior. J Appl Soc Psych 1980; 10 : 436-443

[25] Tanaka H. Swimming exercise: impact of aquatic exercise on cardiovascular health. Sports Med 2009; 39: 377-387

[26] Tanaka H, Bassett DR, Howley ET et al. Swimming training lowers the resting blood pressure in individuals with hypertension. J Hypertens 1997; 15: 651-657

[27] Levy CM, Kolin E, Berson BL. Cross training: risk or benefit? an evaluation of injuries in four athlete populations. Sports Med Clin Forum 1986; 3: 1-8

[28] Alkatan M, Baker JR, Machin DR et al. Improved function and reduced pain after swimming and cycling training in patients with osteoarthritis. J Rheumatol 2016; 43: 666-672

[29] Holmer I. Swimming physiology. Ann Physiol Anthropol 1992; 11: 269-276

[30] Newcomer SC, Leuenberger UA, Hogeman CS et al. Different vasodilator responses of human arms and legs. J Physiol 2004; 556: 1001-1011
[31] Nualnim N, Barnes JN, Tarumi T et al. Comparison of central artery elasticity in swimmers, runners, and the sedentary. Am J Cardiol 2011; 107: 783-787

[32] Rowell LB, O'Leary D, Kellogg DL. Integration of cardiovascular control systems in dynamic exercise. In: Rowell LB, Shephard JT, Eds. Handbook of Physiology, Exercise: Regulation and Integration of Multiple Systems. Bethesda, MD: American Physiological Society; 1996: $770-838$

[33] Tanaka H, Shimizu S, Ohmori F et al. Increases in blood flow and shear stress to nonworking limbs during incremental exercise. Med Sci Sports Exerc 2006; 38: 81-85

[34] Alkatan M, Machin DR, Baker JR et al. Effects of swimming and cycling exercise intervention on vascular function in patients with osteoarthritis. Am J Cardiol 2016; 117: 141-145

[35] Hasegawa N, Fujie S, Horii N et al. Effects of different exercise modes on arterial stiffness and nitric oxide synthesis. Med Sci Sports Exerc 2018; 50: 1177-1185

[36] Nualnim N, Parkhurst K, Dhindsa M et al. Effects of swimming training on blood pressure and vascular function in adults $>50$ years of age. Am J Cardiol 2012; 109: 1005-1010

[37] Aizawa K, Petrella RJ. Acute and chronic impact of dynamic exercise on arterial stiffness in older hypertensives. Open Cardiovasc Med J 2008; 2: $3-8$

[38] Ferrier KE, Waddell TK, Gatzka CD et al. Aerobic exercise training does not modify large-artery compliance in isolated systolic hypertension. Hypertension 2001; 38: 222-226

[39] Seals DR, Tanaka H, Clevenger CM et al. Blood pressure reductions with exercise and sodium restriction in postmenopausal women with elevated systolic pressure: role of arterial stiffness. J Am Coll Cardiol 2001; 38: 506-513

[40] Sherlock L, Fournier S, DeVallancxe E et al. Effects of shallow water aerobic exercise training on arterial stiffness and pulse wave analysis in older individuals. Int J Aquatic Res Educ 2014; 8: 310-320

[41] Scheer AS, Naylor LH, Gan SK et al. The effects of water-based exercise training in people with type 2 diabetes. Med Sci Sports Exerc 2020; 52: 417-424

[42] Suntraluck S, Tanaka H, Suksom D. The relative efficacy of land-based and water-based exercise training on macro- and microvascular functions in older patients with type 2 diabetes. J Aging Phys Act 2017; 25: 446-452

[43] Laurent M, Daline T, Malika B et al. Training-induced increase in nitric oxide metabolites in chronic heart failure and coronary artery disease: An extra benefit of water-based exercises? Eur J Cardiovasc Prev Rehabil 2009; 16: 215-221

[44] Lambert BS, Greene NP, Carradine AT et al. Aquatic treadmill training reduces blood pressure reactivity to physical stress. Med Sci Sports Exerc 2014; 46: 809-816

[45] Parker Jones P, Davy KP, Desouza CA et al. Total blood volume in endurance-trained postmenopausal females: relation to exercise mode and maximal aerobic capacity. Acta Physiol Scand 1999; 166: 327-333

[46] Volianitis S, Yoshiga CC, Secher NH. The physiology of rowing with perspective on training and health. Eur J Appl Physiol 2020; 120: 1943-1963

[47] Petersen SE, Wiesmann F, Hudsmith LE et al. Functional and structural vascular remodeling in elite rowers assessed by cardiovascular magnetic resonance. J Am Coll Cardiol 2006; 48: 790-797

[48] Kawano H, lemitsu M, Gando Y et al. Habitual rowing exercise is associated with high physical fitness without affecting arterial stiffness in older men. J Sports Sci 2012; 30: 241-246

[49] Prasad K, Reriani MK, Murad MH et al. Impact of mind body therapies on vascular endothelial dysfunction and implications for cardiovascular disease management. J Complement Integr Med 2011; 8: 1-13 
[50] Sugawara J, Tarumi T, Tanaka H. Effect of mirthful laughter on vascular function. Am J Cardiol 2010; 106: 856-859

[51] Bressington D, Yu C, Wong W et al. The effects of group-based Laughter Yoga interventions on mental health in adults: A systematic review. J Psychiatr Ment Health Nurs 2018; 25: 517-527

[52] Prakhinkit S, Suppapitiporn S, Tanaka H et al. Effects of Buddhist walking meditation on functional fitness, depression, and endotheliumdependent vasodilation in the elderly with depressive symptoms. J Alternat Complement Med 2014; 20: 411-416

[53] Wang JS, Lan C, Chen SY et al. Tai Chi Chuan training is associated with enhanced endothelium-dependent dilation in skin vasculature of healthy older men. J Am Geriatr Soc 2002; 50: 1024-1030

[54] Arce-Esquivel AA, Ballard JE, Haas BK et al. Effect of tai chi on vascular function among patients with peripheral neuropathy. J Heart Cardiol 2016; 2: 1-6

[55] Lu X, Hui-Chan CW, Tsang WW. Effects of Tai Chi training on arterial compliance and muscle strength in female seniors: A randomized clinical trial. Eur J Prev Cardiol 2013; 20: 238-245

[56] Gainey A, Himathongkam T, Tanaka $\mathrm{H}$ et al. Effects of Buddhist walking meditation on glycemic control and vascular function in patients with type 2 diabetes. Complement Ther Med 2016; 26: 92-97

[57] Wooten SV, Stray-Gundersen S, Tanaka H. Hemodynamic and pressor responses to combination of yoga and blood flow restriction. Int J Sports Med 2020; 41: 759-765

[58] Yamamoto K, Kawano H, Gando Y et al. Poor trunk flexibility is associated with arterial stiffening. Am J Physiol Heart Circ Physiol 2009; 297: H1314-H1318

[59] Miles SC, Chou CC, Lin HF et al. Arterial blood pressure and hemodynamic responses to yoga practice. Alternat Therapies Health Med 2013; 19: 38-45

[60] Cortez-Cooper MY, Anton MM, Devan AE et al. The effects of strength training on central arterial compliance in middle-aged and older adults. Eur J Cardiovasc Prev Rehabil 2008; 15: 149-155

[61] Lawrence MM, Cooley ID, Huet YM et al. Factors influencing isometric exercise training-induced reductions in resting blood pressure. Scand J Med Sci Sports 2015; 25: 131-142

[62] Melville GW, Chang D, Colagiuri B et al. Fifteen minutes of chair-based yoga postures or guided meditation performed in the office can elicit a relaxation response. Evid Based Complement Alternat Med 2012; 2012: 501986

[63] Hunter SD, Tarumi T, Dhindsa M et al. Hatha yoga and vascular function: Results from cross-sectional and interventional studies. J Bodyw Mov Ther 2013; 17: 322-327

[64] Kim S, Bemben MG, Bemben DA. Effects of an 8-month yoga intervention on arterial compliance and muscle strength in premenopausal women. J Sports Sci Med 2012; 11: 322-330

[65] Patil SG, Aithala MR, Das KK. Effect of yoga on arterial stiffness in elderly subjects with increased pulse pressure: A randomized controlled study. Complement Ther Med 2015; 23: 562-569

[66] Hunter SD, Dhindsa MS, Cunningham E et al. The effect of Bikram yoga on endothelial function in young and middle-aged and older adults. J Bodyw Mov Ther 2017; 21: 30-34

[67] Hunter SD, Dhindsa MS, Cunningham E et al. The effect of Bikram yoga on arterial stiffness in young and older adults. J Altern Complement Med 2013; 19: 930-934

[68] Imamura M, Biro S, Kihara T et al. Repeated thermal therapy improves impaired vascular endothelial function in patients with coronary risk factors. J Am Coll Cardiol 2001; 38: 1083-1088

[69] Brunt VE, Jeckell AT, Ely BR et al. Acute hot water immersion is protective against impaired vascular function following forearm ischemia-reperfusion in young healthy humans. Am J Physiol Regul Integr Comp Physiol 2016; 311: R1060-R1067
[70] Hunter SD, Laosiripisan J, Elmenshawy A et al. Effects of yoga interventions practised in heated and thermoneutral conditions on endothelium-dependent vasodilatation: The Bikram yoga heart study. Exp Physiol 2018; 103: 391-396

[71] Pedersen MT, Vorup J, Nistrup A et al. Effect of team sports and resistance training on physical function, quality of life, and motivation in older adults. Scand J Med Sci Sports 2017; 27: 852-864

[72] Schmidt JF, Andersen TR, Andersen LJ et al. Cardiovascular function is better in veteran football players than age-matched untrained elderly healthy men. Scand J Med Sci Sports 2015; 25: 61-69

[73] Schmidt JF, Hansen PR, Andersen TR et al. Cardiovascular adaptations to 4 and 12 months of football or strength training in 65- to 75-year-old untrained men. Scand J Med Sci Sports 2014; 24: 86-97

[74] Krustrup P, Randers MB, Andersen LJ et al. Soccer improves fitness and attenuates cardiovascular risk factors in hypertensive men. Med Sci Sports Exerc 2013; 45: 553-560

[75] Kim JH, Sher S, Wang F et al. Impact of American-style football participation on vascular function. Am J Cardiol 2015; 115: 262-267

[76] Kim JH, Hollowed C, Liu C et al. Weight gain, hypertension, and the emergence of a maladaptive cardiovascular phenotype among US football players. JAMA Cardiol 2019; 4: 1221-1229

[77] Miyachi M, Donato AJ, Yamamoto K et al. Greater age-related reductions in central arterial compliance in resistance-trained men. Hypertension 2003; 41: 130-135

[78] Miyachi M, Kawano H, Sugawara J et al. Unfavorable effects of resistance training on central arterial compliance: a randomized intervention study. Circulation 2004; 110: 2858-2863

[79] Cortez-Cooper MY, DeVan AE, Anton MM et al. Effects of high intensity resistance training on arterial stiffness and wave reflection in women. Am J Hypertens 2005; 18: 930-934

[80] Kawano H, Tanaka H, Miyachi M. Resistance training and arterial compliance: keeping the benefits while minimizing the stiffening. J Hypertens 2006; 24: 1753-1759

[81] Maeda S, Otsuki T, lemitsu M et al. Effects of leg resistance training on arterial function in older men. Br J Sports Med 2006; 40: 867-869

[82] Green DJ, Fowler DT, O'Driscoll JG et al. Endothelium-derived nitric oxide activity in forearm vessels of tennis players. J Appl Physiol (1985) 1996; 81: 943-948

[83] Kagaya A, Ohmori F, Okuyama S et al. Blood flow and arterial vessel diameter change during graded handgrip exercise in dominant and non-dominant forearms of tennis players. Adv Exp Med Biol 2010; 662: 365-370

[84] Sinoway LI, Musch TI, Minotti JR et al. Enhanced maximal metabolic vasodilatation in the dominant forearms of tennis players. J Appl Physiol (1985) 1986; 61: 673-678

[85] Dela F, Niederseer D, Patsch W et al. Glucose homeostasis and cardiovascular disease biomarkers in older alpine skiers. Scand J Med Sci Sports 2011; 21: 56-61

[86] Niederseer D, Steidle-Kloc E, Mayr M et al. Effects of a 12-week alpine skiing intervention on endothelial progenitor cells, peripheral arterial tone and endothelial biomarkers in the elderly. Int J Cardiol 2016; 214: 343-347

[87] Tanaka H. Effects of cross-training. Transfer of training effects on VO2max between cycling, running and swimming. Sports Med 1994; 18: $330-339$

[88] Harriss DJ, Macsween A, Atkinson G. Standards for ethics in sport and exercise science research: 2020 update. Int J Sports Med 2019; 40: 813-817 